\title{
Spherical F-Tilings by Triangles and $r$-Sided Regular Polygons, $r \geq 5$
}

\author{
Catarina P. Avelino * Altino F. Santos ${ }^{\dagger}$ \\ Department of Mathematics \\ UTAD, 5001 - 801 Vila Real, Portugal
}

Submitted: Dec 19, 2007; Accepted: Jan 24, 2008; Published: Feb 4, 2008

Mathematics Subject Classification: 52C20, 52B05, 20B35

\begin{abstract}
The study of dihedral f-tilings of the sphere $S^{2}$ by spherical triangles and equiangular spherical quadrangles (which includes the case of 4-sided regular polygons) was presented in [3]. Also, in [6], the study of dihedral f-tilings of $S^{2}$ whose prototiles are an equilateral triangle (a 3-sided regular polygon) and an isosceles triangle was described (we believe that the analysis considering scalene triangles as the prototiles will lead to a wide family of f-tilings). In this paper we extend these results, presenting the study of dihedral f-tilings by spherical triangles and $r$-sided regular polygons, for any $r \geq 5$. The combinatorial structure, including the symmetry group of each tiling, is given in Table 1.
\end{abstract}

Keywords: dihedral f-tilings, isometric foldings, spherical trigonometry

\section{Introduction}

Let $S^{2}$ be the Euclidean sphere of radius 1. By a dihedral folding tiling (f-tiling, for short) of the sphere $S^{2}$ whose prototiles are a spherical $r$-sided regular polygon, $P^{r}$, and a spherical triangle, $T$, we mean a polygonal subdivision $\tau$ of $S^{2}$ such that each cell (tile) of $\tau$ is congruent to $P^{r}$ or $T$ and the vertices of $\tau$ satisfy the angle-folding relation, i.e., each vertex of $\tau$ is of even valency $2 n, n \geq 2$, and the sums of alternate angles are equal; that is,

$$
\sum_{i=1}^{n} \alpha_{2 i}=\sum_{i=1}^{n} \alpha_{2 i-1}=\pi,
$$

\footnotetext{
* (cavelino@utad.pt) Research Unit CM-UTAD of University of Trás-os-Montes e Alto Douro.

$\dagger$ (afolgado@utad.pt) Supported partially by the Research Unit Mathematics and Applications, through the Foundation for Science and Technology (FCT).
} 
where the angles $\alpha_{i}$ around any vertex of $\tau$ are ordered cyclically. In this paper we shall discuss dihedral f-tilings by spherical triangles and spherical $r$-sided regular polygons $(r \geq 5)$.

F-tilings are intrinsically related to the theory of isometric foldings of Riemannian manifolds, introduced by S. A. Robertson [7] in 1977.

The classification of f-tilings was initiated by Ana Breda [1], with a complete classification of all spherical monohedral f-tilings. Later on, in 2002, Y. Ueno and Y. Agaoka [11] have established the complete classification of all triangular monohedral tilings (without any restrictions on angles).

Dihedral f-tilings by spherical parallelograms and spherical triangles was recently obtained in papers $[3,4,5]$. Robert Dawson has also been interested in special classes of spherical tilings, see $[8,9,10]$ for instance.

The study of dihedral f-tilings of the sphere by triangles and $r$-sided regular polygons was initiated in 2004, [3], where the case $r=4$ was considered. We believe that the case $r=3$ lead to a wide family of f-tilings and it is still in development. We will assume throughout the text that $r \geq 5$.

We shall denote by $\Omega\left(P^{r}, T\right)$ the set, up to an isomorphism, of all dihedral f-tilings of $S^{2}$ whose prototiles are $P^{r}$ and $T$.

From now on $P^{r}(r \geq 5)$ is a $r$-sided regular polygon of internal angle $\alpha$ and edge length $a$ and $T$ is a spherical triangle of internal angles $\beta, \gamma$ and $\delta$, with edge lengths $b$ (opposite to $\beta$ ), $c$ (opposite to $\gamma$ ) and $d$ (opposite to $\delta$ ), see Figure 1.
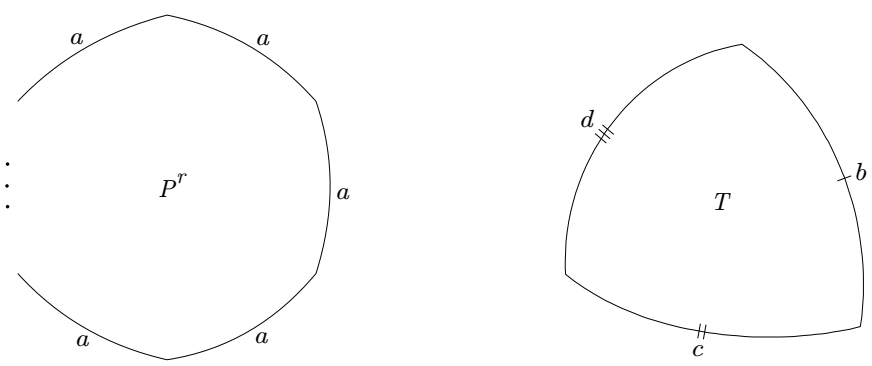

Figure 1: Prototiles: a spherical $r$-sided regular polygon and a spherical triangle

It follows straightway that

$$
\beta+\gamma+\delta>\pi \quad \text { and } \quad \frac{3 \pi}{5} \leq \frac{(r-2) \pi}{r}<\alpha<\pi
$$

In [3] it was established that any $\tau \in \Omega\left(P^{r}, T\right)$ has necessarily vertices of valency four. We shall describe the set $\Omega\left(P^{r}, T\right)$ by considering different cases separately depending on the nature of $T$ (isosceles or scalene). If $T$ is an equilateral spherical triangle, then it is easy to see that $\Omega\left(P^{r}, T\right)=\emptyset$ (the proof is analogous to the case $r=4$ in [3]).

In order to get any dihedral f-tiling $\tau \in \Omega\left(P^{r}, T\right)$, we find useful to start by considering one of its planar representations $(P R)$, beginning with a common vertex to a spherical regular polygon and a spherical triangle in adjacent positions. 
In the diagrams that follows it is convenient to label the tiles according to the following procedures:

(i) The tiles by which we begin the $P R$ of the tiling $\tau \in \Omega\left(P^{r}, T\right)$ are a regular polygon and a triangle in adjacent positions, labelled by 1 and $1^{\prime}$, respectively;

(ii) For $j \geq 2$, the location of tile $j$ can be deduced from the configuration of tiles $\left(1,1^{\prime}, 2,3, \ldots, j-1\right)$ and from the hypothesis that the configuration is part of a complete $P R$ of a f-tiling (except in the cases indicated).

The paper is structured as follows. In Section 2 we obtain the class of all dihedral spherical f-tilings by isosceles triangles, $T$, and $r$-sided regular polygons, $P^{r}$. The case when the prototile $T$ is a scalene triangle is studied in Section 3. The paper is finished in Section 4 with a briefly summary of the f-tilings obtained, where the combinatorial structure of each tiling is presented.

\section{Dihedral Spherical F-Tilings by Isosceles Triangles and $r$-Sided Regular Polygons}

In this section $P^{r}$ and $T$ denote, respectively, a spherical $r$-sided regular polygon $(r \geq 5)$ and a spherical isosceles triangle, where $P^{r}$ has angle $\alpha$, and $T$ has angles $\beta, \gamma, \gamma$, with $\beta \neq \gamma$. As referred before, one has $\frac{(r-2) \pi}{r}<\alpha<\pi$ and $2 \gamma+\beta>\pi$.

Any element of $\Omega\left(P^{r}, T\right)$ has at least two cells congruent, respectively, to $P^{r}$ and $T$, such that they are in adjacent positions and in one of the situations illustrated in Figure 2.
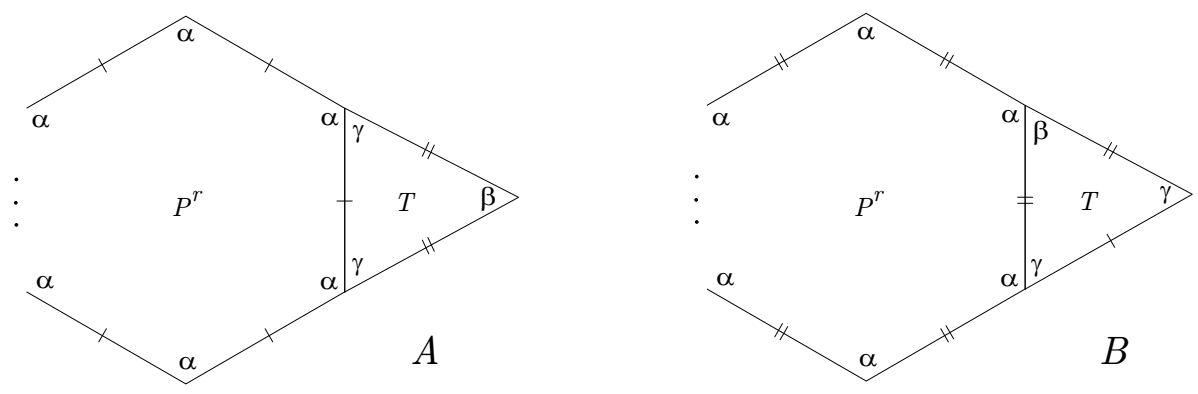

Figure 2: Distinct cases of adjacency

We begin by noting that the edge length $a$ of $P^{r}$ is uniquely determined by $\alpha$ (extension of $[2$, Proposition 3]):

$$
\cos a=\frac{\cos \frac{2 \pi}{r}+\cos ^{2} \frac{\alpha}{2}}{\sin ^{2} \frac{\alpha}{2}}=\frac{1+\cos \alpha+2 \cos \frac{2 \pi}{r}}{1-\cos \alpha} .
$$

The two distinct cases of adjacency illustrated in Figure 2 will be now analyzed separately in Proposition 2.2 and Proposition 2.3, respectively. The next lemma will be useful in such propositions. 
Lemma 2.1 Let $k_{1} \alpha+k_{2} \beta+k_{3} \gamma=\pi$ be a sum of alternate angles around a vertex $v$ of a tiling $\tau \in \Omega\left(P^{r}, T\right)$. Then at least one of the $k_{i}$ is 0 .

Proof. If each $k_{i} \geq 1$, then

$$
\pi=k_{1} \alpha+k_{2} \beta+k_{3} \gamma \geq \alpha+\beta+\gamma>\pi
$$

which is impossible.

Proposition 2.2 Let $P^{r}$ and $T$ be a spherical $r$-sided regular polygon and a spherical isosceles triangle, respectively, such that they are in adjacent positions as illustrated in Figure $2-A$. Then, $\Omega\left(P^{r}, T\right) \neq \emptyset$ iff

$$
\alpha+\gamma=\pi \quad \text { and } \beta=\frac{\pi}{2} \quad \text { or } \quad \alpha+\beta=\pi \quad \text { and } \gamma=\frac{\pi}{2} .
$$

The first case leads to $r=6$ and to a unique f-tiling, denoted by $\mathcal{C}$, with $\alpha=\arccos \frac{-2}{3}$. A planar representation is illustrated in Figure 7. For its $3 D$ representation see Figure 8.

In the second situation, for each $r \geq 5$, there is a single f-tiling given by an antiprism, denoted by $\mathcal{A}_{\alpha}^{r}$, with $\alpha=\arccos \left(1-2 \cos \frac{\pi}{r}\right)=\alpha_{0}^{r}$. A planar representation is illustrated in Figure 10. Some 3D representations are illustrated in Figure 11.

Proof. Suppose that $P^{r}$ and $T$ are in adjacent positions as illustrated below (Figure 3). Consider also that this configuration is contained in some element of $\Omega\left(P^{r}, T\right)$. With the

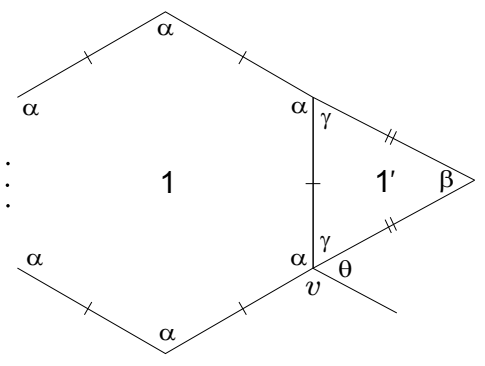

Figure 3: Planar representation

labelling used in Figure 3, we have $\theta \neq \alpha$, and so

$$
\theta=\gamma \quad \text { or } \quad \theta=\beta
$$

We begin by considering the case $\theta=\gamma$.

(i) If $\theta=\gamma$, then necessarily

$$
\alpha+\gamma=\pi \quad \text { or } \quad \alpha+\gamma<\pi .
$$

1. Suppose firstly that $\alpha+\gamma=\pi$. Then the initial $P R$ is extended to get the one illustrated in Figure 4. As $\alpha>\frac{(r-2) \pi}{r}(r \geq 5)$, then $\gamma<\frac{2 \pi}{r}$. And so

$$
\beta>\frac{(r-4) \pi}{r},
$$




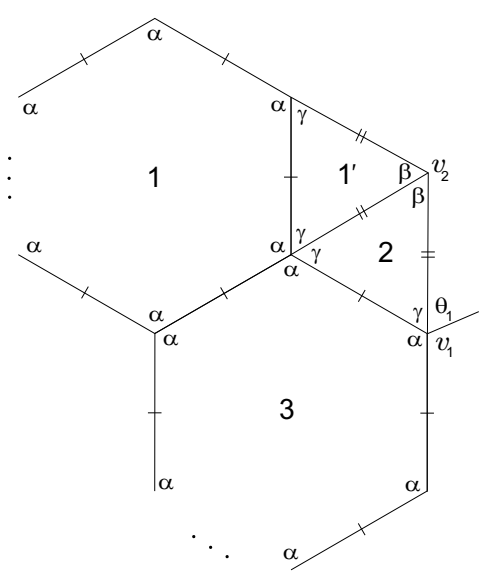

Figure 4: Planar representation

since $2 \gamma+\beta>\pi$. We show that the angle $\theta_{1}$ at vertex $v_{1}$ (Figure 4), adjacent to $\gamma$, is also $\gamma$. Clearly this angle cannot be $\alpha$. If it is $\beta$, then $\alpha+\beta=\pi$, since $\alpha+\beta+\rho>\pi$, for all $\rho \in\{\alpha, \beta, \gamma\}$ (observe that $\alpha+\beta+\beta>\frac{(3 r-10) \pi}{r} \geq \pi$, for any $r \geq 5$ ). Nevertheless $\alpha+\beta=\pi=\alpha+\gamma$ implies $\beta=\gamma$, which is a contradiction. Therefore $\theta_{1}=\gamma$. Using an analogous argument successive times around vertices surrounded by adjacent angles $\alpha$ and $\gamma$ we conclude that vertex $v_{2}$ is exclusively surrounded by angles $\beta$. And so $\beta=\frac{\pi}{k}$, for some $k \geq 2$. As $\beta>\frac{(r-4) \pi}{r}$, then $r \leq 7$.

We will now consider separately the cases $r=5, r=6$ and $r=7$.

If $r=5$, then either $\beta=\frac{\pi}{2}, \beta=\frac{\pi}{3}$ or $\beta=\frac{\pi}{4}$, whose corresponding extended planar representations are illustrated in Figure 5.
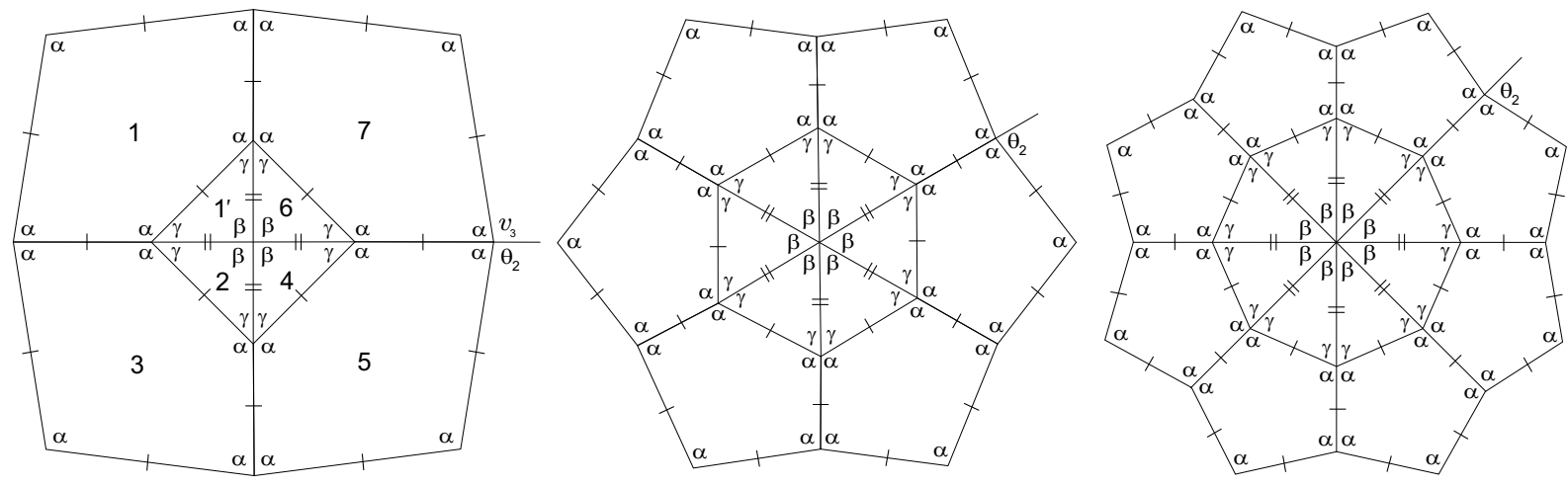

$$
\beta=\frac{\pi}{2}
$$

$\beta=\frac{\pi}{3}$

$$
\beta=\frac{\pi}{4}
$$

Figure 5: Planar representations

Nevertheless all these cases lead to a contradiction. We consider only the first one since the others are analogous. At vertex $v_{3}$ in Figure $5\left(\beta=\frac{\pi}{2}\right)$, the angle $\theta_{2}$ must be $\gamma$. And we get the configuration illustrated in Figure 6. But then we must have $\theta_{3}=\alpha$, which is impossible $(\alpha+\alpha>\pi)$. 


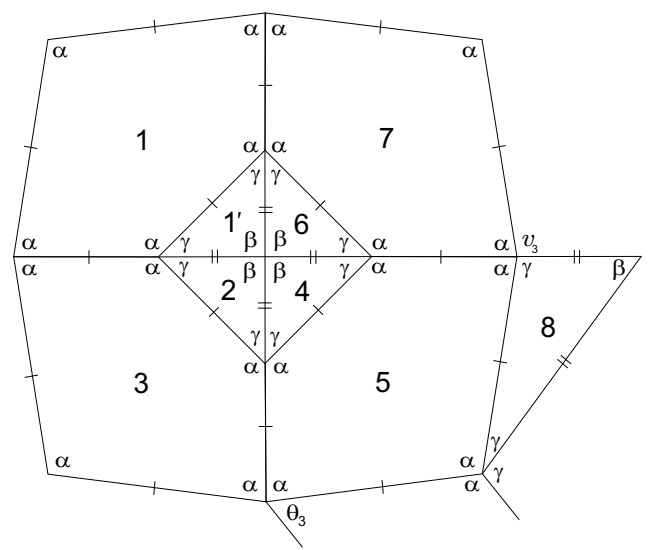

Figure 6: Planar representation

If $r=6$, then $\beta=\frac{\pi}{2}$ and the $P R$ illustrated in Figure 4 (with $r=6$ ) is extended in a unique way to get the configuration represented in Figure 7.

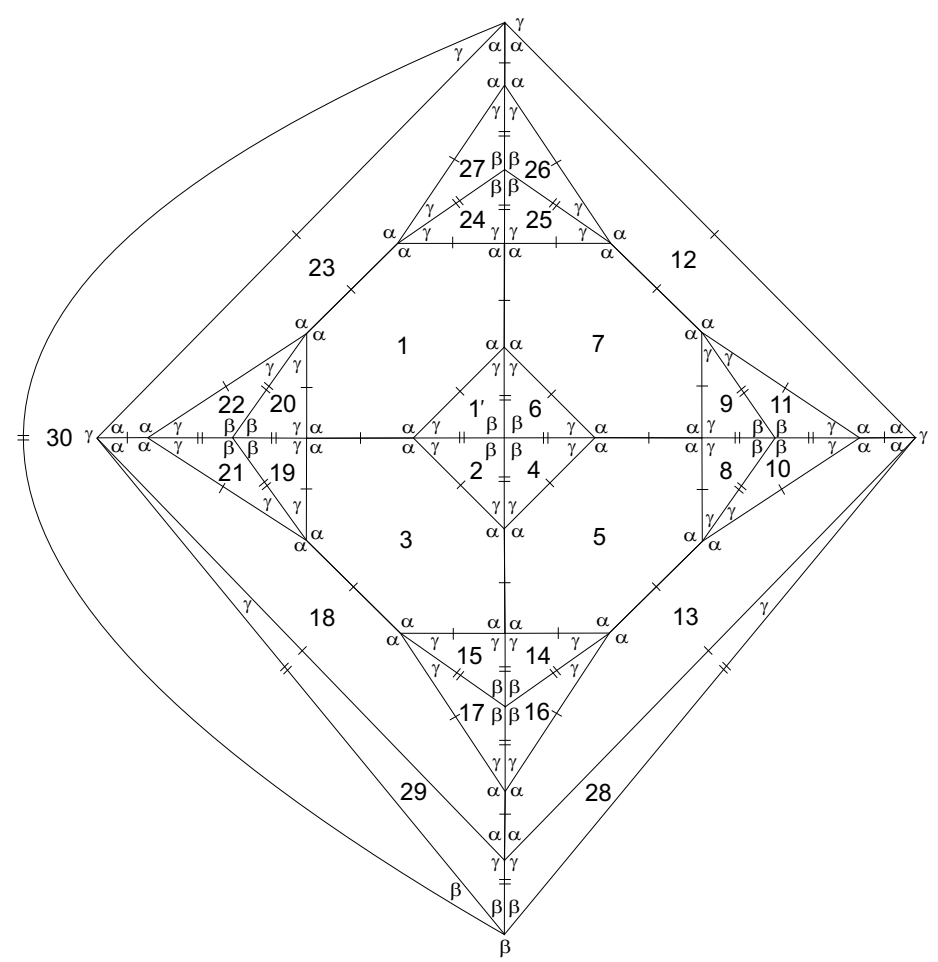

Figure 7: Extended planar representation of $\mathcal{C}$

Now, if $a$ is the edge length of $P^{r}$ (opposite to $\beta$ ), then, as $\gamma \in\left(0, \frac{\pi}{3}\right)$,

$$
\begin{aligned}
& \frac{\cos \frac{\pi}{2}+\cos ^{2} \gamma}{\sin ^{2} \gamma}=\frac{\cos \frac{\pi}{3}+\cos ^{2} \frac{\alpha}{2}}{\sin ^{2} \frac{\alpha}{2}} \Leftrightarrow \\
& \cot ^{2} \gamma=\frac{2-\cos \gamma}{1+\cos \gamma} \quad \Leftrightarrow
\end{aligned}
$$




$$
\cos \gamma=\frac{2}{3}
$$

Hence $\gamma=\arccos \frac{2}{3}$ and $\alpha=\pi-\gamma \approx 131.8^{\circ}$. We shall denote this f-tiling by $\mathcal{C}$. It is a straightforward exercise to show that the edge lengths are $a=b=\arccos \frac{4}{5}$ and $c=\arccos \frac{2 \sqrt{5}}{5}$ (opposite to $\gamma$ ). A 3D representation of $\mathcal{C}$ is given in Figure 8 .

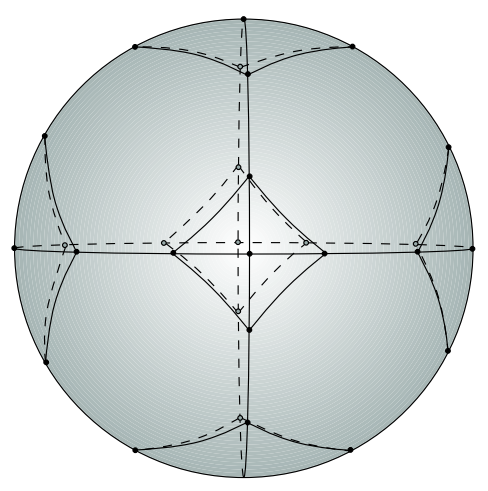

Figure 8: F-tiling $\mathcal{C}$

If $r=7$, then we also have $\beta=\frac{\pi}{2}$, and we reach, as in case $r=5$, to a contradiction (see Figure 9(a), where a vertex with three angles $\alpha$ cannot be avoided).

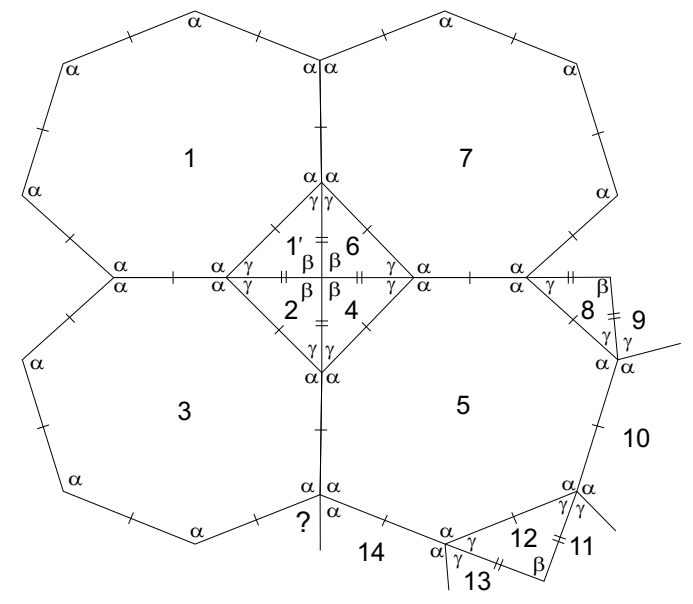

(a)

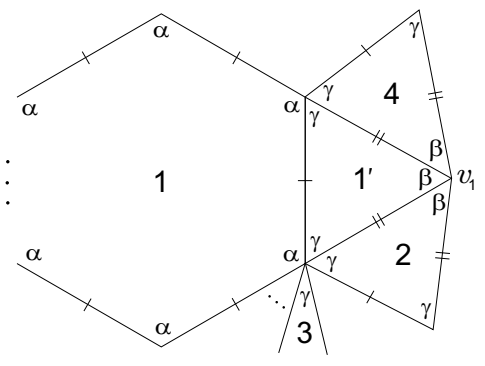

(b)

Figure 9: Planar representations

2. Suppose now that $\alpha+\gamma<\pi$ (Figure 3). In this case we have, by Lemma 2.1, $\alpha+k \gamma=\pi$, for some $k \geq 2$. Thus, tile 4 (Figure 9(b)) is uniquely determined and so 
$\beta \leq \frac{\pi}{2}$ (see vertex $v_{1}$ ). On the other hand, since $\alpha>\frac{(r-2) \pi}{r} \geq \frac{3 \pi}{5}$, we obtain $k \gamma<\frac{2 \pi}{5}$, i.e., $\gamma<\frac{2 \pi}{5 k} \leq \frac{\pi}{5}$. But since $2 \gamma+\beta>\pi$, then $\beta>\frac{3 \pi}{5}>\frac{\pi}{2}$, which is impossible.

(ii) Consider now that $\theta=\beta$ (Figure 3 ). We study separately the cases

$$
\alpha+\beta=\pi \quad \text { and } \quad \alpha+\beta<\pi .
$$

1. Suppose firstly that $\alpha+\beta=\pi$. Taking in account the edge lengths, one gets $\alpha+\beta=\pi=\gamma+\gamma$, and so $\gamma=\frac{\pi}{2}$. The extension of the planar f-tiling is uniquely determined, as illustrated in Figure 10.

Now, since $a$ is the edge length of $P^{r}$ (opposite to $\beta$ ), and using (1), we obtain

$$
\cos a=\frac{1+\cos \alpha+2 \cos \frac{2 \pi}{r}}{1-\cos \alpha}=-\cos \alpha .
$$

Therefore,

$$
\cos ^{2} \alpha-2 \cos \alpha-1-2 \cos \frac{2 \pi}{r}=0 \text { and so } \cos \alpha=1-\sqrt{2+2 \cos \frac{2 \pi}{r}}=1-2 \cos \frac{\pi}{r} .
$$

Taking in account that $\alpha \in\left(\frac{(r-2) \pi}{r}, \pi\right)$, then we conclude that

$$
\alpha=\arccos \left(1-2 \cos \frac{\pi}{r}\right)=\alpha_{0}^{r} .
$$

The edge length of $T$ opposite to $\gamma$ is $c=\frac{\pi}{2}$, for any $r \geq 5$.

The f-tiling with such a $P R$ will be denoted by $\mathcal{A}_{\alpha}^{r}$, with $\alpha=\alpha_{0}^{r}$ (Figure 10). It is

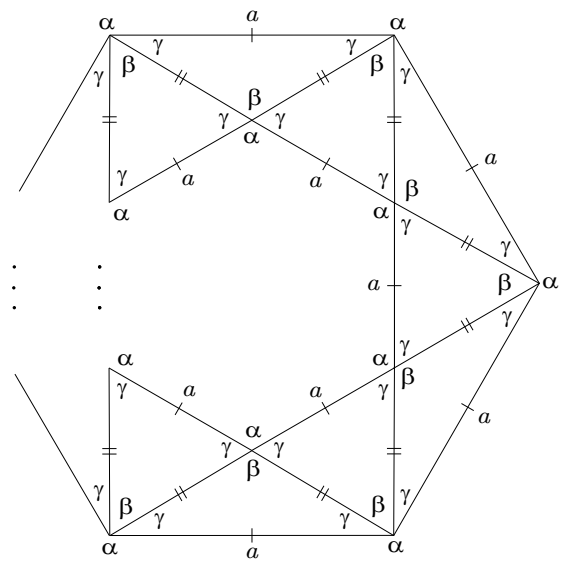

Figure 10: Planar representation of $\mathcal{A}_{\alpha_{0}^{r}}^{r}, \alpha_{0}^{r}=\arccos \left(1-2 \cos \frac{\pi}{r}\right), r \geq 5$

easy to see that $\alpha_{0}^{r}$ is an increasing function in $r$. We have $\alpha_{0}^{5} \approx 128.2^{\circ}, \alpha_{0}^{6} \approx 137.1^{\circ}$ and $\lim _{r \rightarrow+\infty} \alpha_{0}^{r}=\pi$. 3D representations of $\mathcal{A}_{\alpha_{0}^{5}}^{5}$ and $\mathcal{A}_{\alpha_{0}^{6}}^{6}$ are given in Figure 11.

2. Suppose now that $\alpha+\beta<\pi$. Then, by Lemma 2.1, $\alpha+k \beta=\pi$, for some $k \geq 2$. As $\alpha>\frac{(r-2) \pi}{r} \geq \frac{3 \pi}{5}$, then $\beta<\frac{2 \pi}{k r} \leq \frac{\pi}{5}$. And so $\gamma>\frac{2 \pi}{5}$, since $2 \gamma+\beta>\pi$.

According to the edge lengths (Figure 12), the sum of alternate angles containing $\gamma$ is $\gamma+(k-1) \beta+\gamma$, which is greater than $\pi$, and so we reach a contradiction. 

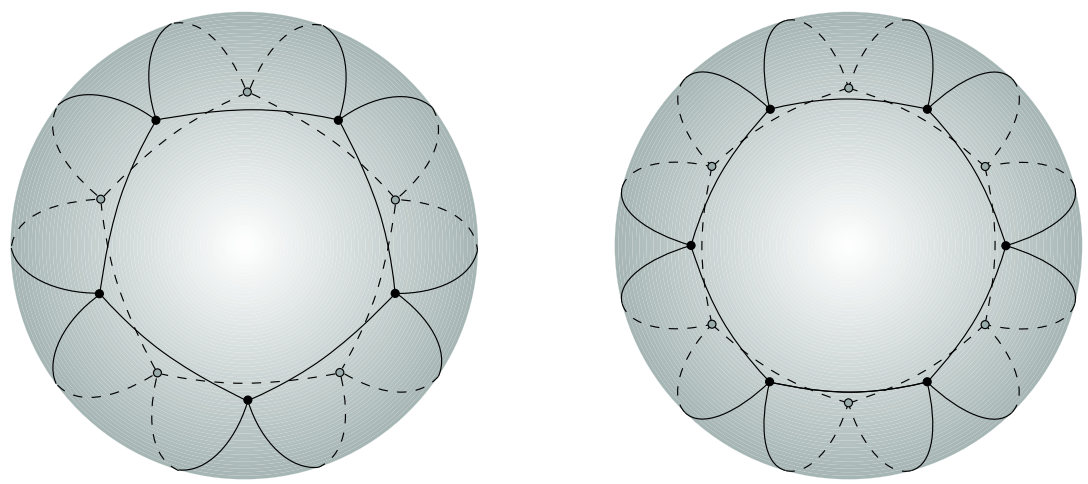

Figure 11: F-tilings $\mathcal{A}_{\alpha_{0}^{r}}^{r}, \alpha_{0}^{r}=\arccos \left(1-2 \cos \frac{\pi}{r}\right)$, cases $r=5$ and $r=6$

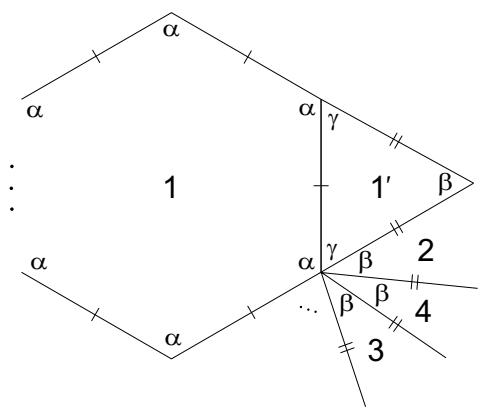

Figure 12: Planar representation

Proposition 2.3 Let $P^{r}$ and $T$ be a r-sided regular polygon and an isosceles triangle, respectively, such that they are in adjacent positions as illustrated in Figure $2-B$. Then, $\Omega\left(P^{r}, T\right) \neq \emptyset$ iff $r=5, \alpha+\gamma=\pi$ and $\beta=\alpha$. In this situation, there is a single tiling given by an antiprism $\mathcal{A}_{\alpha}^{5}$, with $\alpha=\frac{4 \pi}{5}$. A planar representation and a $3 D$ representation of $\mathcal{A}_{\frac{4 \pi}{5}}^{5}$ are given in Figure 14(a) and Figure 14(b), respectively.

Proof. Let $v$ be a common vertex to adjacent tiles, congruent to $P^{r}$ and $T$, respectively, and surrounded by $\alpha$ and $\gamma$. The configuration of a such f-tiling near $v$ must be the one illustrated in Figure 13.

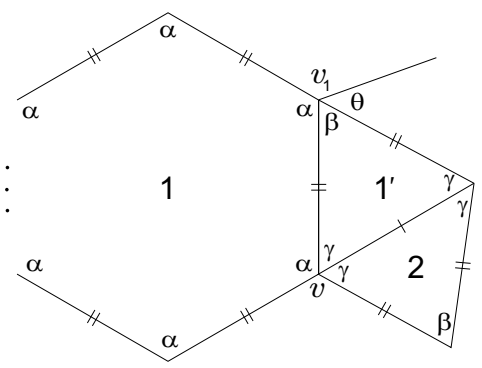

Figure 13: Planar representation 
At vertex $v$ we must have

$$
\alpha+\gamma=\pi \quad \text { or } \quad \alpha+\gamma<\pi
$$

(i) Suppose that $\alpha+\gamma=\pi$. With the labelling of Figure $13, \theta=\beta$ or $\theta=\gamma$. If $\theta=\beta$, then $\alpha+\beta<\pi$ (otherwise $\beta=\gamma$ ). And so, by Lemma 2.1, $\alpha+k \beta=\pi$, for some $k \geq 2$. Hence, $\beta<\frac{2 \pi}{k r} \leq \frac{\pi}{5}$. Therefore, $2 \gamma+\beta<\frac{4 \pi}{r}+\frac{\pi}{5} \leq \pi$, which is a contradiction. We conclude that $\theta=\gamma$ and vertex $v_{1}$ is surrounded by the cyclic sequence of angles $(\alpha, \beta, \gamma, \gamma)$, with $\alpha+\gamma=\pi=\beta+\gamma$, and so $\alpha=\beta$.

The extension of the planar f-tiling is now uniquely determined. As

$$
-\frac{\cos \alpha}{1-\cos \alpha}=\frac{1+\cos \alpha+2 \cos \frac{2 \pi}{r}}{1-\cos \alpha}
$$

we may conclude that

$$
\cos \alpha=-\frac{1}{2}-\cos \frac{2 \pi}{r} \quad \text { and so } \quad r=5
$$

observe that if $r>5$, then $-\frac{1}{2}-\cos \frac{2 \pi}{r}<-1$. Therefore, $\cos \alpha=-\frac{1}{2}-\cos \frac{2 \pi}{5}=-\frac{1+\sqrt{5}}{4}$ and so $\alpha=\frac{4 \pi}{5}=\beta$.

The edge lengths of the prototiles of $\mathcal{A}_{\frac{4 \pi}{5}}^{5}$ are $a=c=\arccos \frac{\sqrt{5}}{5}$ and $b=\arccos \frac{-\sqrt{5}}{5}$. A planar representation of $\mathcal{A}_{\frac{4 \pi}{5}}^{5}$ is given in Figure 14(a). Its 3D representation is illustrated in Figure 14(b).

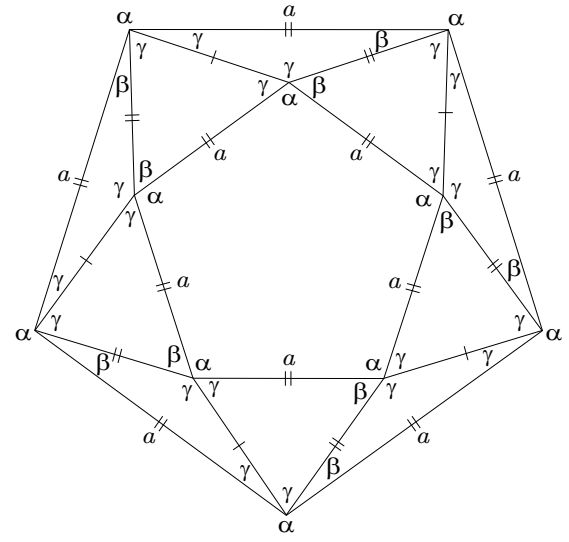

(a) Planar representation

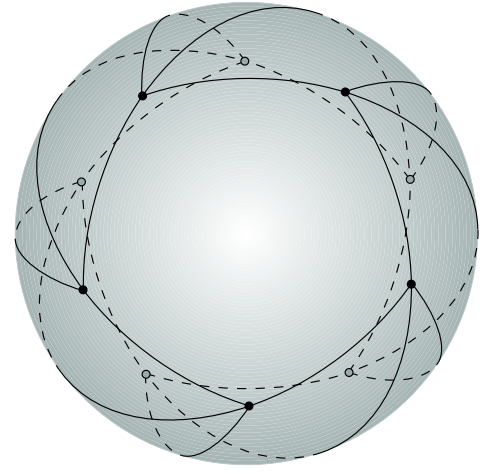

(b) $3 \mathrm{D}$ representation

Figure 14: F-tiling $\mathcal{A}_{\frac{4 \pi}{5}}^{5}$

(ii) Suppose now that $\alpha+\gamma<\pi$. Thus, by Lemma 2.1, $\alpha+k \gamma=\pi$, with $k \geq 2$. And so $\beta>\alpha>\gamma$, since $\beta+2 \gamma>\pi$. It follows that the sum of alternate angles containing $\beta$ at vertex $v_{1}$ (Figure 13) cannot be defined, which is impossible. 


\section{Dihedral Spherical F-Tilings by Scalene Triangles and $r$-Sided Regular Polygons}

Here $T$ stands for a scalene spherical triangle of angles $\beta, \gamma$ and $\delta$, with $\beta>\gamma>\delta$ $(\beta+\gamma+\delta>\pi)$, and $P^{r}(r \geq 5)$ is a spherical $r$-sided regular polygon of angle $\alpha$ and side a.

If $\tau \in \Omega\left(P^{r}, T\right)$, then there are necessarily two cells of $\tau$ congruent to $P^{r}$ and $T$, respectively, such that they are in adjacent positions and in one of the situations illustrated in Figure 15.

The three distinct cases of adjacency will be now analyzed in Proposition 3.1, Proposi-
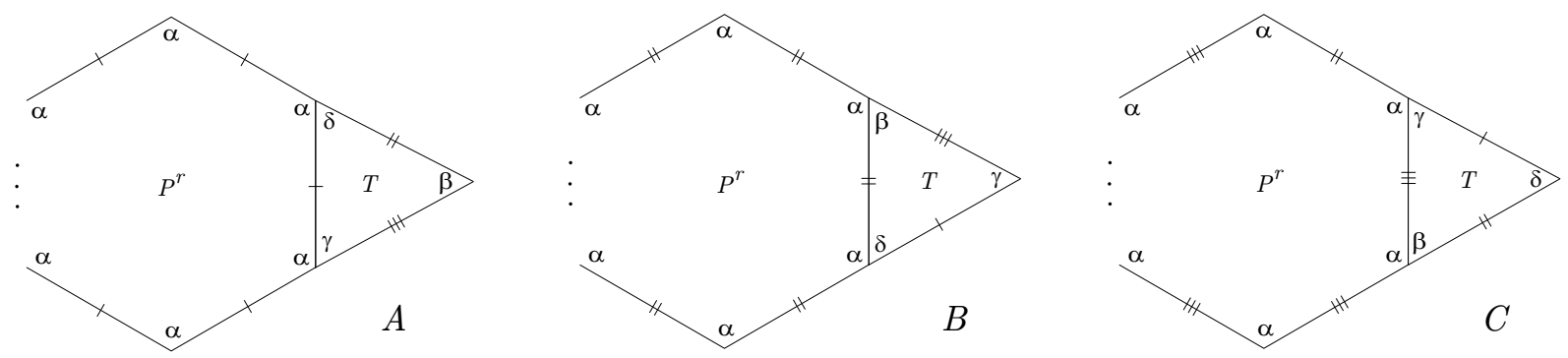

Figure 15: Distinct cases of adjacency

tion 3.2 and Proposition 3.3, respectively.

Proposition 3.1 Let $\tau \in \Omega\left(P^{r}, T\right)$. Then $\tau$ does not contain a pair of tiles in the position of Figure 15-A.

Proof. Suppose that $\tau \in \Omega\left(P^{r}, T\right)$ has two cells in adjacent positions as illustrated in Figure 15- $A$. Let $\theta_{1}$ be the angle adjacent to $\gamma$ and opposite to $\alpha$ and let $\theta_{2}$ be the angle adjacent to $\delta$ and opposite to $\alpha$ as indicated in Figure 16(a). It follows that

$$
\theta_{1} \in\{\beta, \gamma\} \quad \text { and } \quad \theta_{2} \in\{\beta, \delta\}
$$

If $\theta_{1}=\beta$, then $\alpha+\beta=\pi$ (otherwise, $\alpha+\beta+\rho \geq \alpha+\beta+\delta>\pi$, for all $\rho \in\{\alpha, \beta, \gamma, \delta\}$ ). Taking in account the edge lengths, we obtain $\alpha+\beta=\pi=\gamma+\delta<\pi$, which is a contradiction.

Thus, $\theta_{1}=\gamma$ and analogously we obtain $\theta_{2}=\delta$. Consequently $\beta=\frac{\pi}{2}$ and a corresponding $P R$ is illustrated in Figure 16(b).

Since $\alpha>\beta$, we have, at vertex $v_{1}, \alpha+\gamma=\pi$. Consequently, at vertex $v_{2}$, we obtain $\alpha+k \delta=\pi$, for some $k \geq 2$. As $\beta+\gamma+\delta>\pi, \beta=\frac{\pi}{2}$ and $\gamma=k \delta, k \geq 2$, we obtain $\gamma>\frac{\pi}{3}$. On the other hand, since $\alpha>\frac{(r-2) \pi}{r}$, we have $\gamma<\frac{2 \pi}{r}$. And so $r=5$.

Now, $\frac{\pi}{2}<\gamma+\delta<\frac{2 \pi}{5}+\frac{2 \pi}{5 k}$, and so

$$
k=2 \text { or } \quad k=3 \text {. }
$$




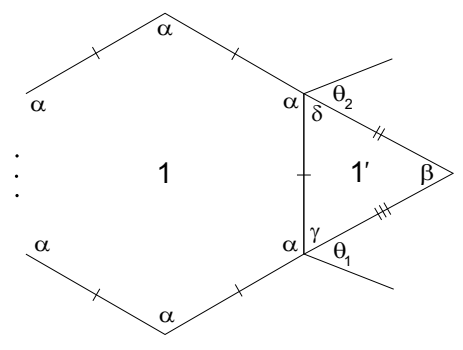

(a)

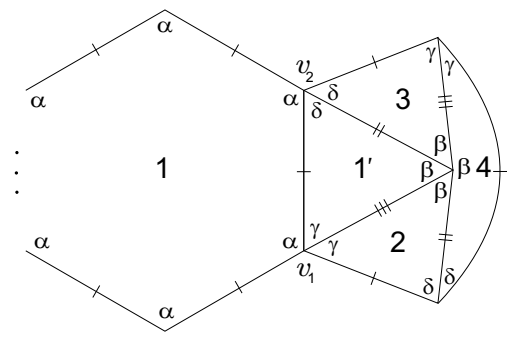

(b)

Figure 16: Planar representations

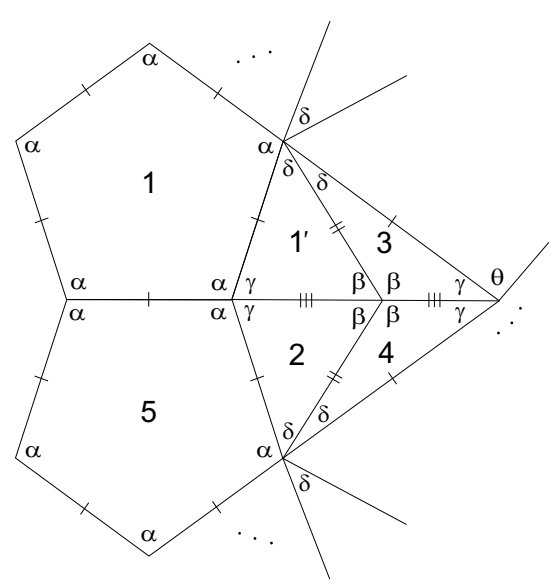

(a)

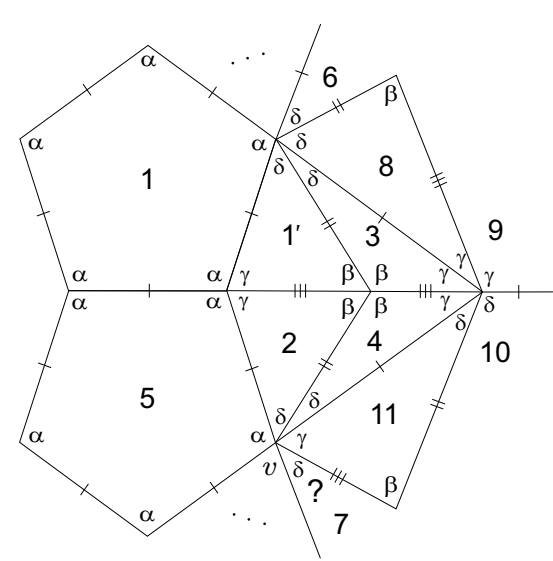

(b)

Figure 17: Planar representations

With the labelling used in Figure 17(a), the angle $\theta$ must be $\alpha$. In fact, the analysis of the edge lengths implies $\theta \neq \beta$ and $\theta \neq \delta$. If $\theta=\gamma$, then $\gamma+\gamma+\delta=\pi$ and consequently we reach a contradiction at vertex $v$ in Figure 17(b) (see edge lengths of tile 7 ). Thus, $\theta=\alpha$ and we obtain the $P R$ below (Figure 18).

We study now the cases $k=2$ and $k=3$ separately.

We shall consider firstly that $k=3$, i.e., $\alpha+3 \delta=\pi$. The $P R$ illustrated in Figure 18 is extended to get the one represented in Figure 19 (observe that, after we set up tile 17, we get $2 \gamma<\pi$, and so $2 \gamma+\delta=\pi$, at vertex $w_{1}$ ). Taking in account the edge lengths, we have no way to have the angle folding relation full filled at vertex $w_{2}$.

Consider now the case $k=2$. With the labelling of Figure $18, \theta^{\prime}=\delta$ or $\theta^{\prime}=\gamma$. If $\theta^{\prime}=\delta$, then we obtain the $P R$ illustrated in Figure 20, where a vertex surrounded by the cyclic sequence of angles $(\alpha, \alpha, \delta, \delta, \gamma, \gamma)$ takes place, leading to a contradiction. The case $\theta^{\prime}=\gamma$ is similar and also leads to a contradiction. 


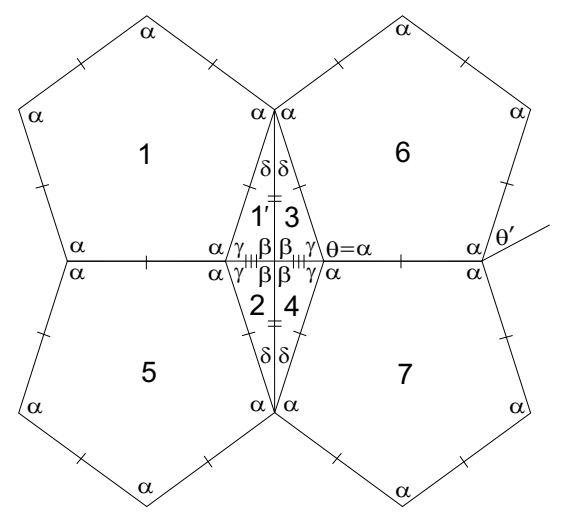

Figure 18: Planar representation

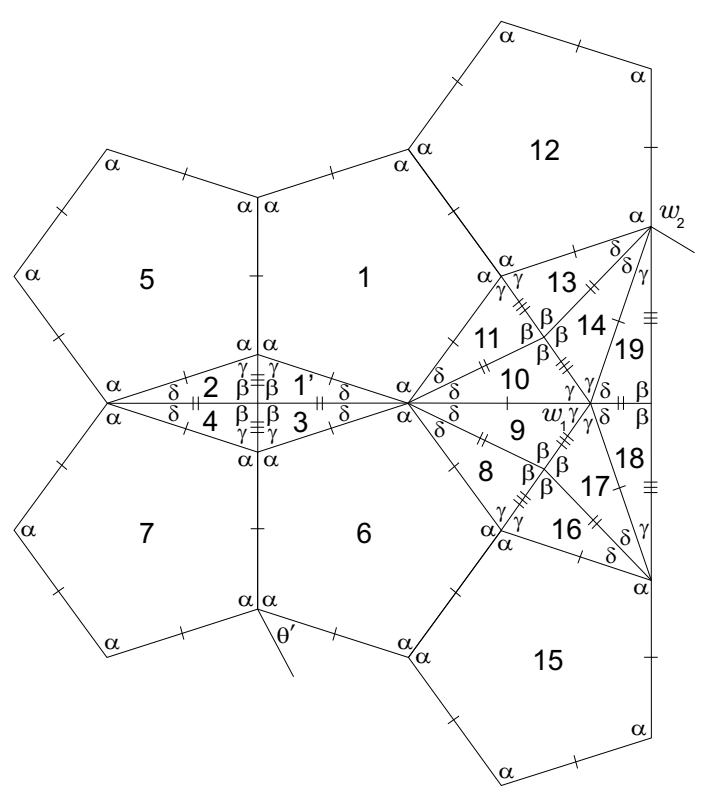

Figure 19: Planar representation

Proposition 3.2 Let $\tau \in \Omega\left(P^{r}, T\right)$ and suppose that $P^{r}$ and $T$ are in adjacent positions as illustrated in Figure 15-B. Then, $\tau$ is an antiprism $\mathcal{A}_{\alpha}^{r}$, where $\alpha+\gamma=\pi=\beta+\delta$, $r=5$ and $\alpha \in\left(\frac{4 \pi}{5}, \pi\right)$.

Proof. Suppose that there are two cells in adjacent positions as illustrated in Figure 15- $B$. Let $\theta$ be the angle adjacent to $\beta$ and opposite to $\alpha$ (Figure 21(a)). It follows that

$$
\theta=\beta \quad \text { or } \quad \theta=\gamma
$$

(i) If $\theta=\beta$, then necessarily $\alpha+\beta=\pi$ and the cyclic sequence of angles around $v$ is $(\alpha, \beta, \beta, \alpha)$. Now, the vertices surrounded by $\alpha$ and $\delta$ cannot have valency 4 , and so $\alpha+k \delta=\pi$, for some $k \geq 2$. A vertex surrounded by, at least, four consecutive angles $\gamma$ 


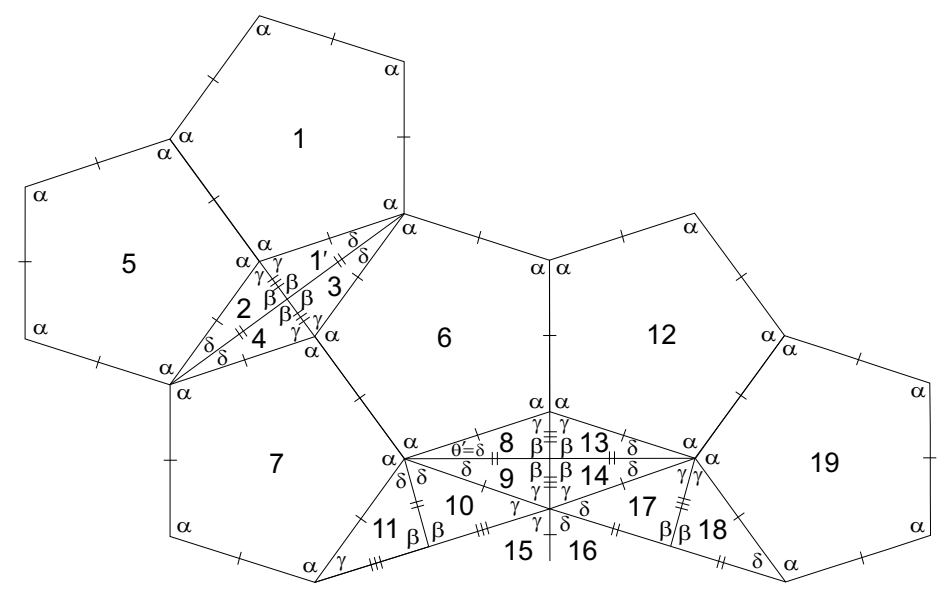

Figure 20: Planar representation

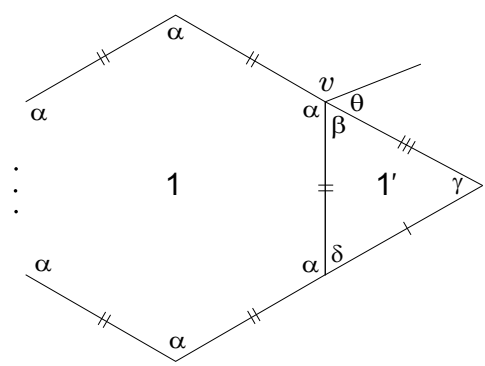

(a)

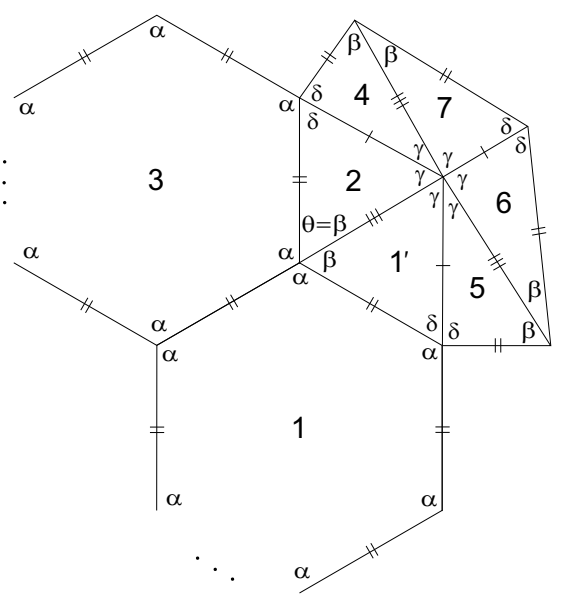

(b)

Figure 21: Planar representations

takes place. Taking in account the edge lengths, we conclude that $\gamma=\frac{\pi}{3}$, as represented in Figure 21(b).

Observe now that if $r \geq 6$, then $\alpha>\frac{2 \pi}{3}$, and so $\beta<\frac{\pi}{3}=\gamma$, which is impossible. Therefore $r=5$. In this case, $\beta<\frac{2 \pi}{5}$, and so $\delta>\frac{4 \pi}{15}$, since $\beta+\gamma+\delta$ has to be greater than $\pi$. On the other hand, we have $\beta=k \delta(k \geq 2)$, and so $\delta<\frac{\pi}{5}$, which is a contradiction.

(ii) Suppose now that $\theta=\gamma$. We consider separately the cases

$$
\alpha+\gamma=\pi \quad \text { and } \quad \alpha+\gamma<\pi .
$$

1. If $\alpha+\gamma=\pi$, beginning the $P R$ from vertex $v$ (Figure 21(a)) and taking in account 
the edge lengths of $P^{r}$ and $T$, we conclude that an extended $P R$ of such $\mathrm{f}$-tiling(s) corresponds to antiprism(s) $\tau=\mathcal{A}_{\alpha}^{r}$ such that $\alpha+\gamma=\pi=\beta+\delta$. We will see that, under these angles relations, we have necessarily $r=5$. The refereed $P R$ is shown in Figure 22(a).

Having in consideration the relations between angles, we have $\beta>\alpha>\frac{\pi}{2}, \gamma=\pi-\alpha$ and $\delta=\pi-\beta$.

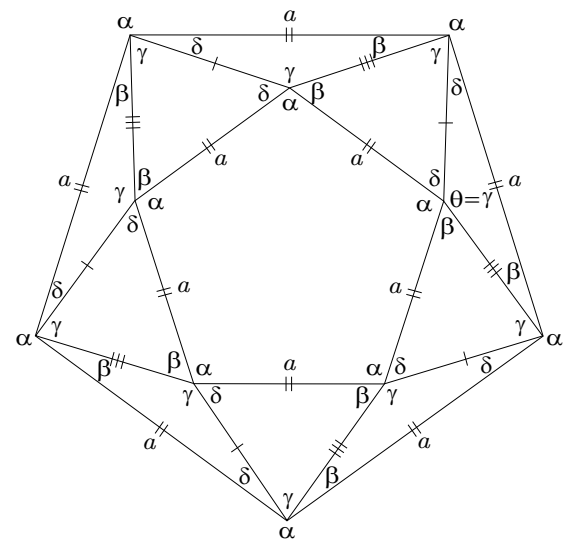

(a) Planar representation

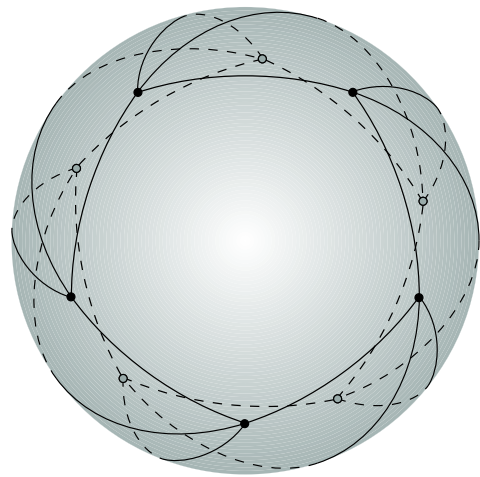

(b) $3 \mathrm{D}$ representation

Figure 22: $\mathcal{A}_{\alpha}^{5}, \alpha \in\left(\frac{4 \pi}{5}, \pi\right)$

One has

$$
\cos a=\frac{1+\cos \alpha+2 \cos \frac{2 \pi}{r}}{1-\cos \alpha}=-\frac{\cos \alpha+\cos ^{2} \beta}{\sin ^{2} \beta},
$$

where $a$ is the edge length of $T$ opposite to $\gamma$. As $\alpha, \beta \in\left(\frac{\pi}{2}, \pi\right)$, we have

$$
\begin{aligned}
\frac{1+\cos \alpha+2 \cos \frac{2 \pi}{r}}{1-\cos \alpha}+\frac{\cos \alpha+\cos ^{2} \beta}{\sin ^{2} \beta} & =0 & \\
\left(\frac{1+\cos \alpha+2 \cos \frac{2 \pi}{r}}{1-\cos \alpha}-1\right) \sin ^{2} \beta & =-\cos \alpha-1 & \Leftrightarrow \\
\sin ^{2} \beta & =\frac{\sin ^{2} \alpha}{-2\left(\cos \alpha+\cos \frac{2 \pi}{r}\right)} & \Leftrightarrow \\
\sin \beta & =\frac{\sin \alpha}{\sqrt{-2\left(\cos \alpha+\cos \frac{2 \pi}{r}\right)}} . &
\end{aligned}
$$

Taking in account that $\beta>\alpha$, we obtain 


$$
\begin{aligned}
\sin \beta & <\sin \alpha & \Leftrightarrow \\
\sqrt{-2\left(\cos \alpha+\cos \frac{2 \pi}{r}\right)} & >1 & \Leftrightarrow \\
\cos \alpha & <-\frac{1}{2}-\cos \frac{2 \pi}{r}, &
\end{aligned}
$$

leading us to conclude that $r=5$ (otherwise, last inequality does not make sense), and so $\alpha \in\left(\frac{4 \pi}{5}, \pi\right)$. Using (3), we may also conclude that $\frac{4 \pi}{5}<\beta<\pi$ and

$$
\lim _{\alpha \rightarrow \frac{4 \pi}{5}} \beta=\frac{4 \pi}{5} \text { and } \lim _{\alpha \rightarrow \pi} \beta=\pi \text {. }
$$

In Figure 22(b) is illustrated a 3D representation of $\mathcal{A}_{\alpha}^{5}, \frac{4 \pi}{5}<\alpha<\pi$.

2. If $\alpha+\gamma<\pi$ (Figure 21(a)), we must have

$$
\beta>\alpha>\gamma>\delta
$$

Taking in account that there are necessarily vertices of valency four, it follows that $\beta+\delta=\pi$ or $\beta+\gamma=\pi$.

If $\beta+\delta=\pi$ then, taking in account the edge lengths, we obtain, at vertex $v$, $\beta+\delta=\pi=\gamma+\alpha<\pi$, which is impossible. It follows that $\beta+\gamma=\pi$ and the sums of alternate angles at vertex $v$ must be $\beta+k \delta=\pi=\alpha+\gamma+(k-1) \delta$, for some $k \geq 2$, as represented in Figure 23 .

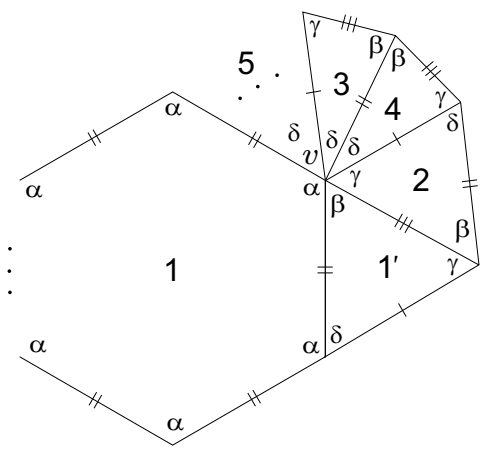

Figure 23: Planar representation

The current conditions between angles allows to write $\alpha, \beta$ and $\gamma$ as functions of $\delta$ :

$$
\alpha=\pi-(2 k-1) \delta, \quad \beta=\pi-k \delta, \quad \gamma=k \delta, \quad k \geq 2 .
$$

Next we prove that these relations between angles lead to a contradiction. In fact, as $\alpha>\frac{(r-2) \pi}{r}$, we have $\delta<\frac{2 \pi}{r(2 k-1)}$.

Since

$$
\frac{1+\cos \alpha+2 \cos \frac{2 \pi}{r}}{1-\cos \alpha}=\frac{\cos \gamma+\cos \beta \cos \delta}{\sin \beta \sin \delta}
$$


we conclude that

$$
\frac{1-\cos ((2 k-1) \delta)+2 \cos \frac{2 \pi}{r}}{1+\cos ((2 k-1) \delta)}=\frac{\cos (k \delta)(1-\cos \delta)}{\sin (k \delta) \sin \delta} .
$$

Considering the (continuously differentiable) function $\psi$ defined in $\left(0, \frac{2 \pi}{r(2 k-1)}\right)$ by

$$
\psi(\delta)=\frac{1-\cos ((2 k-1) \delta)+2 \cos \frac{2 \pi}{r}}{1+\cos ((2 k-1) \delta)}-\frac{\cos (k \delta)(1-\cos \delta)}{\sin (k \delta) \sin \delta},
$$

we have that $\psi$ has no zeros in $\left(0, \frac{2 \pi}{r(2 k-1)}\right)$. Indeed, the first derivative of $\psi$ is

$$
\psi^{\prime}(\delta)=(2 k-1) \cos ^{2} \frac{\pi}{r} \sin ((2 k-1) \delta) \sec ^{4} \frac{(2 k-1) \delta}{2}+\frac{1}{2} \phi(\delta) \csc (\delta) \csc ^{2}(k \delta) \tan \frac{\delta}{2},
$$

where $\phi(\delta)=2 k \sin \delta-\sin (2 k \delta)$. As $0<\delta<2 k \delta<\frac{4 k \pi}{r(2 k-1)}<\pi$ and using the fact that the cosine is a decreasing function on $(0, \pi)$, we conclude that

$$
\phi^{\prime}(\delta)=2 k(\cos \delta-\cos (2 k \delta))>0, \forall \delta \in\left(0, \frac{2 \pi}{r(2 k-1)}\right) \subset(0, \pi) .
$$

Then $\phi$ is an increasing function and since $\phi(0)=0$, we obtain $\phi(\delta)>0$ in $\left(0, \frac{2 \pi}{r(2 k-1)}\right)$. Thus we can easily observe that $\psi^{\prime}(\delta)$ is positive and so $\psi$ is an increasing continuous function in $\left(0, \frac{2 \pi}{r(2 k-1)}\right)$. Since $\lim _{\delta \rightarrow 0^{+}} \psi(\delta)=\cos \frac{2 \pi}{r}-\frac{1}{2 k}>0(k \geq 2)$, we obtain $\psi(\delta)>0$, for all $\delta \in\left(0, \frac{2 \pi}{r(2 k-1)}\right)$, and so $\psi$ has no zeros in $\left(0, \frac{2 \pi}{r(2 k-1)}\right)$.

Proposition 3.3 Suppose that $P^{r}$ and $T$ are in adjacent positions as illustrated in Figure 15-C. Then, in this situation, $\Omega\left(P^{r}, T\right) \neq \emptyset$ iff $\alpha+\delta=\pi=\beta+\gamma$. It is composed by a family of antiprisms $\mathcal{A}_{\alpha}^{r}$, with $\alpha \in\left(\alpha_{0}^{5}, \frac{4 \pi}{5}\right)$ if $r=5$, and $\alpha \in\left(\alpha_{0}^{r}, \pi\right)$ if $r \geq 6$, where $\alpha_{0}^{r}=\arccos \left(1-2 \cos \frac{\pi}{r}\right)$.

Proof. Let $v$ be a common vertex to adjacent tiles, congruent to $P^{r}$ and $T$, and surrounded by $\alpha$ and $\beta$. The $P R$ near $v$ is illustrated in Figure 24(a). With the labelling of this Figure, we must have $\theta=\beta$ or $\theta=\delta$.

(i) Consider firstly that $\theta=\beta$. Then necessarily $\alpha+\beta=\pi$, implying $\alpha>\beta>\gamma>\delta$. Consequently vertex $v_{1}$ (Figure 24(b)) is not of valency four, and so $\alpha+k \delta=\pi$, for some $k \geq 2$.

Taking in account the edge lengths of $P^{r}$ and $T$, the sum of alternate angles containing $\gamma$ at vertex $v_{1}$ is $\gamma+(k-1) \delta+\beta$, which is greater than $\pi$.

(ii) Consider now that $\theta=\delta$ (Figure 24(a)). If $\alpha+\delta<\pi$, then it is a straightforward exercise to show that the sum of alternate angles containing $\beta$ at vertex $v$ cannot be defined (see edge lengths of $P^{r}$ and $T$ ), which is an impossibility. And so $\alpha+\delta=\pi$. The $P R$ represented in Figure 24(a) is now extended in a unique way as illustrated in Figure 25, which represents an antiprism, where

$$
\alpha+\delta=\beta+\gamma=\pi \text {, with } \alpha>\beta>\frac{\pi}{2}>\gamma>\delta .
$$




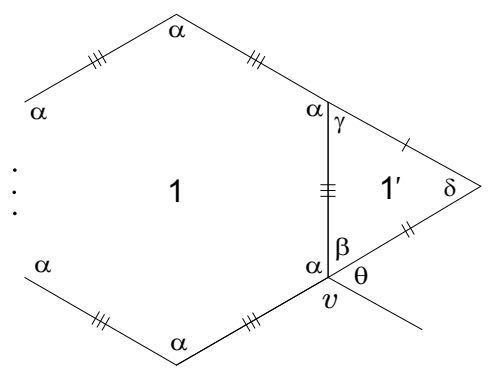

(a)

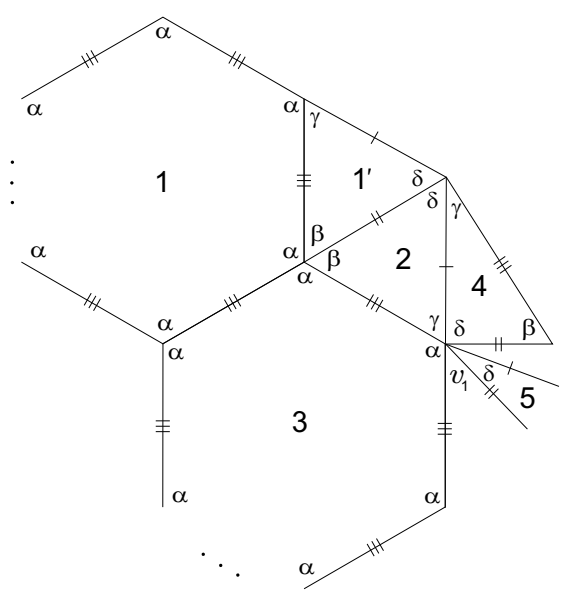

(b)

Figure 24: Planar representations

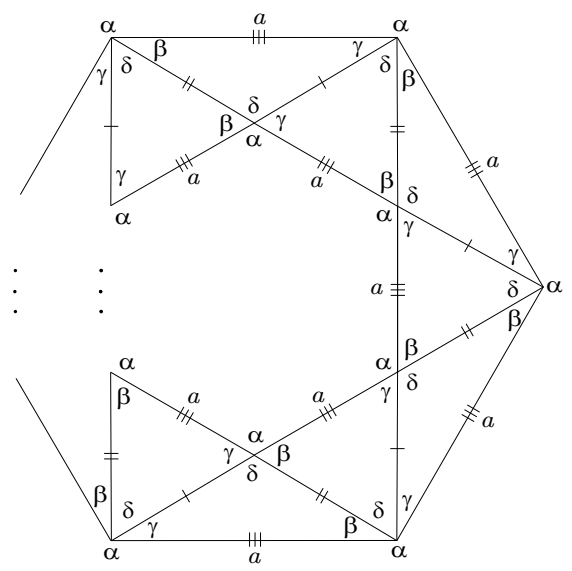

Figure 25: Planar representation of $\mathcal{A}_{\alpha}^{r}, \alpha \in\left(\alpha_{0}^{5}, \frac{4 \pi}{5}\right)$ if $r=5$, and $\alpha \in\left(\alpha_{0}^{r}, \pi\right)$ if $r \geq 6$

As seen before, the equalities (2) and (3) are verified. Using the equality (3), it follows that $\beta<\alpha$ iff $\cos \alpha>-\frac{1}{2}-\cos \frac{2 \pi}{r}$. This condition is always verified if $r \geq 6$. And, if $r=5$, then we have $\beta<\alpha$ iff $\alpha<\frac{4 \pi}{5}$.

We also have $0<\sin \alpha<\sqrt{-2\left(\cos \alpha+\cos \frac{2 \pi}{r}\right)}$. Consequently

$$
\sin ^{2} \alpha<-2 \cos \alpha-2 \cos \frac{2 \pi}{r}
$$

and so

$$
\alpha>\alpha_{0}^{r}=\arccos \left(1-2 \cos \frac{\pi}{r}\right), \quad r \geq 5 .
$$

$3 \mathrm{D}$ representations of $\mathcal{A}_{\alpha}^{5}, \alpha \in\left(\arccos \frac{1-\sqrt{5}}{2}, \frac{4 \pi}{5}\right)$, and $\mathcal{A}_{\alpha}^{6}, \alpha \in(\arccos (1-\sqrt{3}), \pi)$, are 

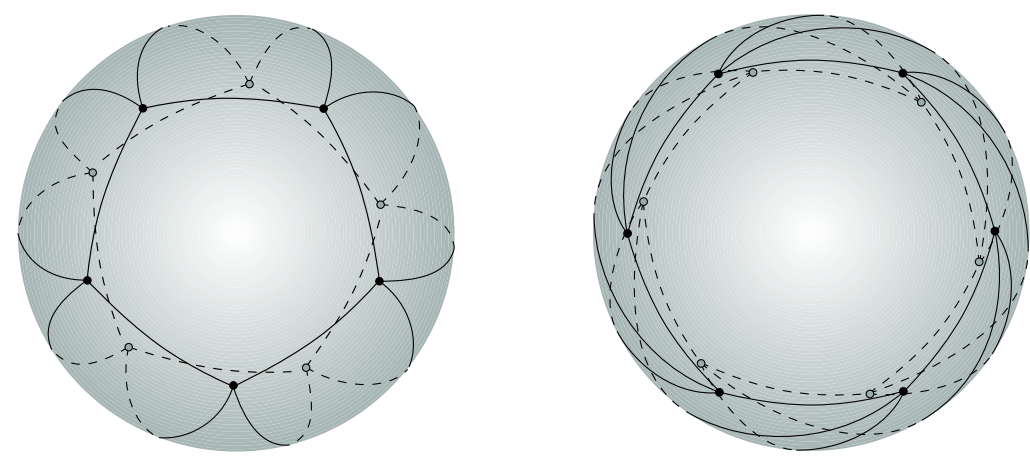

Figure 26: $\mathcal{A}_{\alpha}^{5}, \alpha \in\left(\arccos \frac{1-\sqrt{5}}{2}, \frac{4 \pi}{5}\right)$ and $\mathcal{A}_{\alpha}^{6}, \alpha \in(\arccos (1-\sqrt{3}), \pi)$

illustrated in Figure 26.

With the notation used in the paper, we have:

- if $\alpha \rightarrow\left(\alpha_{0}^{r}\right)^{+}$, then $\beta \rightarrow\left(\frac{\pi}{2}\right)^{+}$and $\gamma \rightarrow\left(\frac{\pi}{2}\right)^{-}$, for any $r \geq 5$;

- if $r=5$ and $\alpha \rightarrow\left(\frac{4 \pi}{5}\right)^{-}$, then $\beta \rightarrow\left(\frac{4 \pi}{5}\right)^{-}$, with $\alpha>\beta$, and so $\gamma \rightarrow\left(\frac{\pi}{5}\right)^{+}, \delta \rightarrow\left(\frac{\pi}{5}\right)^{+}$;

- if $r=5$ and $\alpha \rightarrow\left(\frac{4 \pi}{5}\right)^{+}$, then $\beta \rightarrow\left(\frac{4 \pi}{5}\right)^{+}$, with $\alpha<\beta$, and so $\gamma \rightarrow\left(\frac{\pi}{5}\right)^{-}, \delta \rightarrow\left(\frac{\pi}{5}\right)^{-}$;

- if $r>5$ and $\alpha \rightarrow \pi^{-}$, then $\beta \rightarrow \pi^{-}$, with $\alpha>\beta$, and so $\gamma \rightarrow 0^{+}, \delta \rightarrow 0^{+}$;

\section{Summary}

In Table 1 is shown a complete list of all spherical dihedral f-tilings whose prototiles are an isosceles triangle $T$ of angles $\beta, \gamma, \gamma(\beta \neq \gamma)$ or a scalene triangle $T$ of angles $\beta, \gamma, \delta$ $(\beta>\gamma>\delta)$, and a $r$-sided regular polygon $P^{r}(r \geq 5$; for $r=4$ see [3]) with angle $\alpha$. Our notation is as follows:

- $\alpha_{0}^{r}=\arccos \left(1-2 \cos \frac{\pi}{r}\right), r \geq 5$;

- $\beta=\beta_{\alpha}^{r}, r \geq 5$, is the solution of equation (3);

- $|V|$ is the number of distinct classes of congruent vertices;

- $M$ and $N$ are, respectively, the number of triangles congruent to $T$ and the number of $r$-sided regular polygons congruent to $P^{r}$, used in the dihedral f-tilings;

- $G(\tau)$ is the symmetry group of each tiling $\tau \in \Omega\left(P^{r}, T\right)$; by $D_{n}$ we mean the dihedral group of order $2 n$; the octahedral group is $O_{h} \cong C_{2} \times S_{4}$ (the symmetry group of the cube). 


\begin{tabular}{|c|c|c|c|c|c|c|c|c|}
\hline F-Tiling & $\boldsymbol{\alpha}$ & $\boldsymbol{\beta}$ & $\boldsymbol{\gamma}$ & $\boldsymbol{\delta}$ & $|V|$ & $M$ & $N$ & $G(\tau)$ \\
\hline \hline $\mathcal{A}_{\boldsymbol{\alpha}_{0}^{r}}^{\boldsymbol{r}}, \boldsymbol{r} \geq \mathbf{5}$ & $\alpha_{0}^{r}$ & $\pi-\alpha_{0}^{r}$ & $\frac{\pi}{2}$ & - & 1 & $2 r$ & 2 & $D_{2 r}$ \\
\hline $\mathcal{A}_{\boldsymbol{\alpha}}^{\mathbf{5}}$ & $\left(\alpha_{0}^{5}, \frac{4 \pi}{5}\right)$ & $\beta_{\alpha}^{5}$ & $\pi-\beta_{\alpha}^{5}$ & $\pi-\alpha$ & 1 & 10 & 2 & $D_{5}$ \\
\hline $\mathcal{A}_{\frac{4 \pi}{5}}^{5}$ & $\frac{4 \pi}{5}$ & $\frac{4 \pi}{5}$ & $\frac{\pi}{5}$ & - & 1 & 10 & 2 & $D_{5}$ \\
\hline $\mathcal{A}_{\boldsymbol{\alpha}}^{\mathbf{5}}$ & $\left(\frac{4 \pi}{5}, \pi\right)$ & $\beta_{\alpha}^{5}$ & $\pi-\alpha$ & $\pi-\beta_{\alpha}^{5}$ & 1 & 10 & 2 & $D_{5}$ \\
\hline $\mathcal{A}_{\boldsymbol{\alpha}}^{\boldsymbol{r}}, \boldsymbol{r} \geq \mathbf{6}$ & $\left(\alpha_{0}^{r}, \pi\right)$ & $\beta_{\alpha}^{r}$ & $\pi-\beta_{\alpha}^{r}$ & $\pi-\alpha$ & 1 & $2 r$ & 2 & $D_{r}$ \\
\hline $\mathcal{C}(\boldsymbol{r}=\mathbf{6})$ & $\arccos \frac{-2}{3}$ & $\frac{\pi}{2}$ & $\pi-\alpha$ & - & 2 & 24 & 8 & $O_{h}$ \\
\hline
\end{tabular}

Table 1: The Combinatorial Structure of the Dihedral F-Tilings of $S^{2}$ by $r$-Sided Regular Polygons, $r \geq 5$, and Triangles

In Figure 27 we illustrate in $3 \mathrm{D}$ the dihedral f-tilings obtained in the paper. They consist of:

- a continuous family of pentagonal antiprisms $\left(\mathcal{A}_{\alpha}^{5}\right)_{\alpha \in\left[\alpha_{0}^{5}, \pi\right)}$, in which $T$ is isosceles iff $\alpha=\alpha_{0}^{5}$ or $\alpha=\frac{4 \pi}{5}$; in Figure 27 we have considered $\alpha_{0}^{5}<\alpha_{1}<\frac{4 \pi}{5}<\alpha_{2}<\pi$ and $\alpha_{0}^{5}=\arccos \left(1-2 \cos \frac{\pi}{5}\right)=\arccos \frac{1-\sqrt{5}}{2}$;

- for each $r \geq 6$, a continuous family of antiprisms $\left(\mathcal{A}_{\alpha}^{r}\right)_{\alpha \in\left[\alpha_{0}^{r}, \pi\right)}$, in which $T$ is isosceles iff $\alpha=\alpha_{0}^{r}$; we illustrate $\mathcal{A}_{\alpha_{0}^{6}}^{6}$ and $\mathcal{A}_{\alpha}^{6}$, with $\alpha_{0}^{6}<\alpha<\pi$;

- an sporadic f-tiling $\mathcal{C}$ in which the prototiles are a 6 -sided regular polygon of angle $\alpha=\arccos \frac{-2}{3}$ and an isosceles triangle of angles $\frac{\pi}{2}, \pi-\alpha$ and $\pi-\alpha$.

\section{References}

[1] A. M. Breda, A Class of Tilings of $S^{2}$, Geometriae Dedicata, 44 (1992), 241-253.

[2] A. M. Breda and A. F. Santos, Well centered sherical quadrangles, Beiträge zur Algebra und Geometrie, 44 (2003), 539-549.

[3] A. M. Breda and A. F. Santos, Dihedral f-tilings of the sphere by spherical triangles and equiangular well-centered quadrangles, Beiträge zur Algebra und Geometrie, 45 (2004), 447-461.

[4] A. M. Breda and A. F. Santos, Dihedral f-tilings of the sphere by rhombi and triangles, Discrete Math. Theorical Computer Sci., 7 (2005), 123-140.

[5] A. M. Breda and A. F. Santos, Dihedral f-tilings of the sphere by triangles and well-centered quadrangles, Hiroshima Math. J., 36 (2006), 235-288.

[6] A. M. Breda, P. S. Ribeiro and A. F. Santos, A class of spherical dihedral f-tilings, 2007, submitted. 

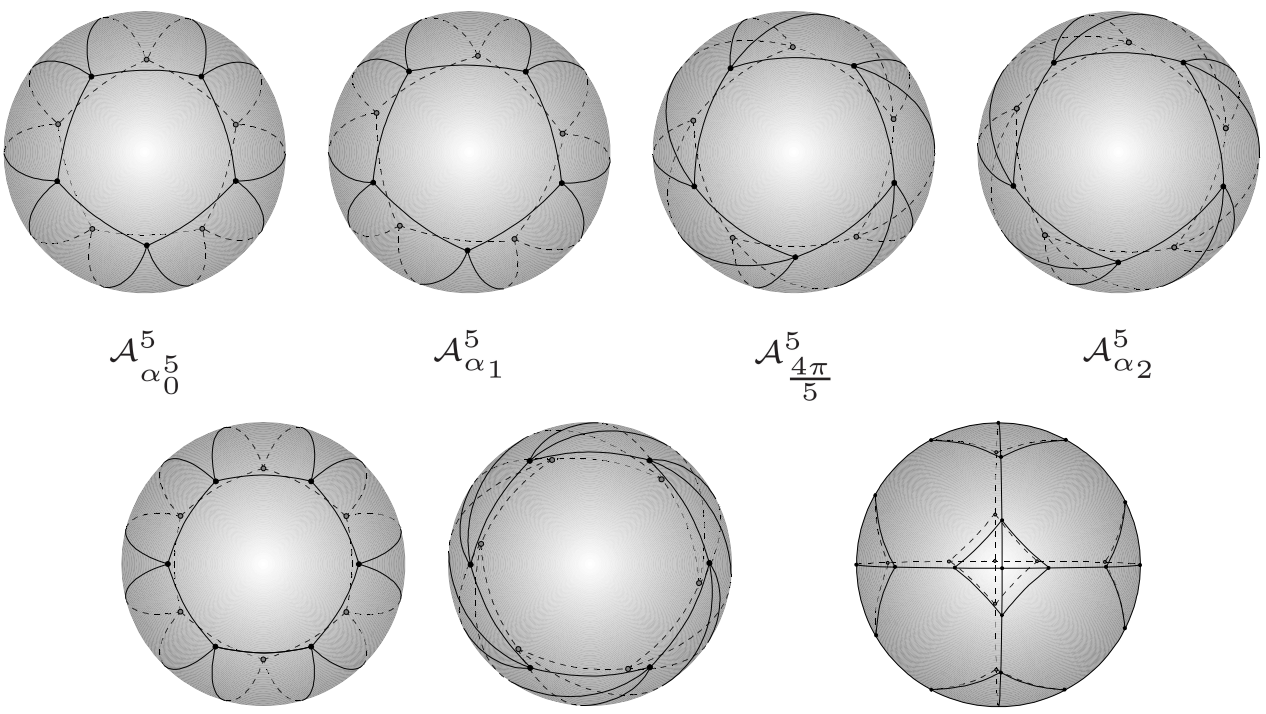

$\mathcal{A}_{\alpha}^{r}, r \geq 6 \quad \mathcal{A}_{\alpha}^{r}, r \geq 6$

$\mathcal{C}$

Figure 27: Dihedral F-Tilings of $S^{2}$ by $r$-Sided Regular Polygons $(r \geq 5)$ and Triangles

[7] S. A. Robertson, Isometric folding of Riemannian manifolds, Proceedings of the Royal Society of Edinburgh, 79 (1977), 275-284.

[8] R. J. Dawson, Tilings of the sphere with isosceles triangles, Disc. and Comp. Geom., 30 (2003), 467-487.

[9] R. J. Dawson and B. Doyle, Tilings of the sphere with right triangles I: the asymptotically right families, Electronic Journal of Combinatorics, 13 (2006), \#R48.

[10] R. J. Dawson and B. Doyle, Tilings of the sphere with right triangles II: the $(1,3,2)$, $(0,2, n)$ subfamily, Electronic Journal of Combinatorics, 13 (2006), \#R49.

[11] Y. Agaoka and Y. Ueno, Classification of tilings of the 2-dimensional sphere by congruent triangles, Hiroshima Math. J., 32 (2002), 463-540. 